\title{
Diversity performance of dual-antenna handsets near operator tissue
}

Michael A. Jensen

jensen@byu.edu

Bruce M. Green

Follow this and additional works at: https://scholarsarchive.byu.edu/facpub

Part of the Electrical and Computer Engineering Commons

\section{Original Publication Citation}

Green, B. M., and M. A. Jensen. "Diversity Performance of Dual-Antenna Handsets Near Operator Tissue." Antennas and Propagation, IEEE Transactions on 48.7 (2): 117-24

\section{BYU ScholarsArchive Citation}

Jensen, Michael A. and Green, Bruce M., "Diversity performance of dual-antenna handsets near operator tissue" (2000). Faculty Publications. 593.

https://scholarsarchive.byu.edu/facpub/593 


\title{
Diversity Performance of Dual-Antenna Handsets Near Operator Tissue
}

\author{
Bruce M. Green and Michael A. Jensen, Member, IEEE
}

\begin{abstract}
This paper presents a computational and experimental study of the diversity performance of two dual-antenna handsets operating indoor in the 902-928 MHz industrial, scientific, and medical (ISM) band. Of particular interest is the effect of the operator tissue on the diversity operation. Key indicators of diversity gain such as branch mean effective gain (MEG) and envelope correlation coefficient are obtained from finite-difference time-domain (FDTD) method simulations as well as from experimental measurements in three different indoor environments. Diversity gain for the handsets is also measured directly. Reasonable agreement is observed between the experimental and simulated results, with both approaches indicating that while the tissue lowers the MEG of individual branches by 3-5 dB, it has little influence on the handset overall diversity performance.
\end{abstract}

Index Terms-Diversity methods, multipath interference.

\section{INTRODUCTION}

I N THE wireless communications industry, a major challenge facing system designers involves the minimization of signal loss during a communications transaction. This is particularly true for links involving mobile terminals such as handsets in environments characterized by severe multipath fading effects. This rapid fading signature can significantly reduce signal reliability and therefore impacts system power requirements, data rates, and frequency utilization.

Several techniques exist for reducing the detrimental effects of multipath fading. One such strategy involves the utilization of multiple antenna elements, configured either as a "smart" antenna or as a simple diversity system to reduce the frequency and severity of fades in the received signal envelope. A large volume of experimental and theoretical research has recently appeared demonstrating the effectiveness of two-antenna diversity on mobile handsets [1]-[10]. These research findings generally suggest that considerable performance improvements can be obtained using even the simplest of diversity combining schemes.

Despite the volume of literature appearing on the subject of antenna diversity, very little work has considered the influence of the operator tissue on handset antenna diversity performance [11]. This paper reports the results of one such study in the indoor propagation environment. The results focus on computational predictions and experimental measurements of branch mean effective gain (MEG), envelope correlation coefficient $\left(\rho_{e}\right)$ and diversity gain for two unique dual-antenna handset configurations operating in the $902-928 \mathrm{MHz}$ industrial, scientific, and medical (ISM) band. MEG and $\rho_{e}$ are computed using

Manuscript received April 15, 1999; revised January 10, 2000

The authors are with the Department of Electrical and Computer Engineering, Brigham Young University, Provo, UT 84602 USA.

Publisher Item Identifier S 0018-926X(00)06929-5. gain patterns from finite-difference time-domain (FDTD) [12] simulations of handsets placed near realistic models of human tissue [13], [14]. Experimental measurements are obtained from a two-channel receiver system connected to prototype handset models. It is shown that the computational and experimental results compare relatively well for MEG observations, while they demonstrate discrepancies for observations of correlation coefficients. The study also reveals that while the tissue reduces the MEG of each branch by 3-5 dB, it exercises little influence on the correlation coefficient and on the measured dual-antenna diversity gain.

\section{HANDSET ANTEnNa Design}

Assessing the diversity performance of realistic dual-antenna handsets operating near human tissue requires the design, fabrication, and testing of different practical antenna/handset geometries. Fig. 1 shows the two configurations considered during this study. The first design consists of two quarter-wavelength monopole antennas, with one mounted on the handset top and another lying on a hinged mouthpiece at the bottom of the handset. The second geometry utilizes the same mouthpiece-mounted monopole structure, but replaces the top-mounted antenna with an integrated planar-inverted $\mathrm{F}$ antenna (PIFA) on the handset back. For simplicity of simulation and consistency in the data collection, the mouthpiece-mounted antennas are positioned to be perpendicular to the handset chassis, as shown in Fig. 1.

The FDTD simulation approach [8], [12] was used to aid in the design of the prototype handset antennas. In these simulations, the handset chassis was assumed to be perfectly conducting and the geometry was modeled using cubical cells of dimension $6.56 \mathrm{~mm}$ per side. Each individual radiating element was designed to provide a return loss $\left|S_{11}\right|$ below $-10 \mathrm{~dB}$ over the entire 902-928 MHz ISM band.

The final antenna designs were mounted on handset cases composed of aluminum and copper. The monopole antenna was fabricated by stripping the outer conductor away from a quarterwavelength section of semi-rigid 0.141 -in $(3.58 \mathrm{~mm})$ coaxial cable and extending the exposed inner conductor through a hole in the top plate of the handset chassis. The outer conductor was then soldered to this top plate. The mouthpiece-mounted antennas were printed on a 0.100 -in $(2.54 \mathrm{~mm})$ thick layer of unclad printed circuit board material $\left(\epsilon_{r}=3.2\right)$ and were fed with a connectorized length of the semi-rigid cable inserted from the back of the handset chassis. The PIFA consisted of a thin plate of copper attached to the handset by means of the shorting plate [see Fig. 1(b)] as well as a thin piece of styrofoam under the 


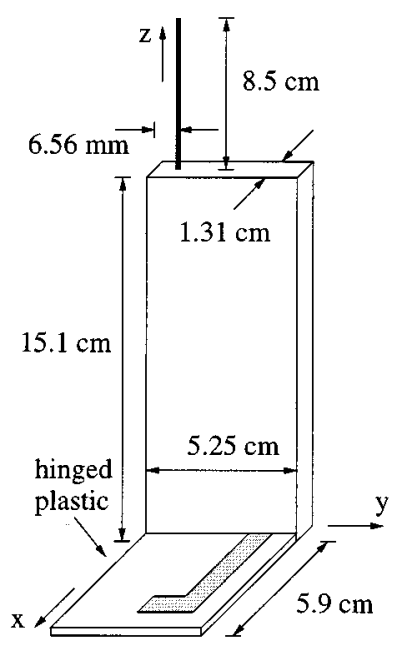

(a)

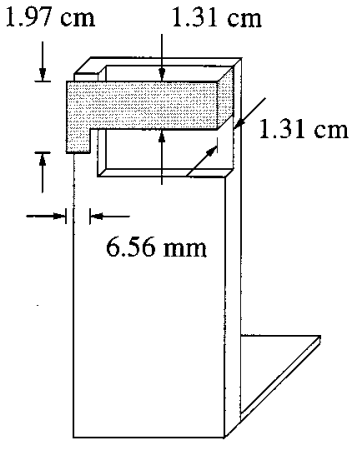

(b)
Fig. 1. Two dual-antenna diversity handset configurations. (a) Monopole /mouthpiece monopole. (b) PIFA/mouthpiece monopole.

plate to provide increased rigidity. The feeding was again accomplished using the semi-rigid coaxial cable.

Testing of the final antenna designs was accomplished using a calibrated Hewlett-Packard 8720B network analyzer. In each case, the variation of the measured input impedance with frequency agreed very well with the simulated predictions with all designs easily satisfying the $-10-\mathrm{dB}$ return-loss specification.

\section{COMPutATional ASSESSMENT}

The first step in assessing the diversity performance of dualantenna handsets involves computation of the antenna MEG and $\rho_{e}$ values. The ability to successfully simulate such parameters allows the designer to investigate diversity performance without costly prototype fabrication and experimental data collection. For these simulations, the FDTD algorithm was used to obtain the radiation pattern for each handset-mounted antenna with the second radiator terminated in a matched load. In all cases, the frequency was chosen to be at the band center $(915 \mathrm{MHz})$ and the handset was positioned at an angle of $60^{\circ}$ from vertical to represent realistic operation.

To evaluate the handset performance when held by the operator, detailed models of the human head and hand were used within the FDTD simulations. In this case, the conducting handset body was located one FDTD cell $(6.56 \mathrm{~mm})$ from the user head. The hand model was centered along the handset and was implemented to wrap around the back and sides of the chassis. Details concerning the implementation of these tissue models can be found in [13].

\section{A. Mean Effective Gain}

The MEG [15], [16] of a handset antenna radiating near a human operator depends on the antenna gain pattern as well as the multipath propagation environment in which it operates. It is defined as

$$
\mathrm{MEG}=G_{e}=\frac{P_{\mathrm{rec}}}{S_{\theta}+S_{\phi}}
$$

where $S_{\theta}$ and $S_{\phi}$ represent the average power contained in the $\hat{\theta}$ and $\hat{\phi}$ components of the incident field (with respect to the antenna coordinate system), respectively, along a random route of the handset. The term $P_{\text {rec }}$ refers to the average power received by the antenna along this route. In order to compute $G_{e}$ for a given configuration, we express the received power using the integral

$$
\begin{aligned}
P_{\text {rec }}=\int_{0}^{2 \pi} \int_{0}^{\pi}[ & S_{\theta} G_{\theta}(\theta, \phi) P_{\theta}(\theta, \phi) \\
& \left.+S_{\phi} G_{\phi}(\theta, \phi) P_{\phi}(\theta, \phi)\right] \sin \theta d \theta d \phi
\end{aligned}
$$

where $G_{\theta}$ and $G_{\phi}$ represent the antenna power gain patterns for the $\hat{\theta}$ and $\hat{\phi}$ polarizations. $P_{\theta}$ and $P_{\phi}$ refer to the angular density of plane waves polarized in the $\hat{\theta}$ and $\hat{\phi}$ directions in the environment. Substituting (2) into (1) yields the result

$$
\begin{aligned}
G_{e}= & \frac{1}{1+X} \int_{0}^{2 \pi} \int_{0}^{\pi} \\
& \cdot\left[X G_{\theta}(\theta, \phi) P_{\theta}(\theta, \phi)+G_{\phi}(\theta, \phi) P_{\phi}(\theta, \phi)\right] \sin \theta d \theta d \phi
\end{aligned}
$$

where $X$ denotes the cross-polarization discrimination ratio (XPR) defined as $X=S_{\theta} / S_{\phi}$.

For these computations, $G_{\theta}$ and $G_{\phi}$ are obtained using the FDTD approach for the antenna/handset/tissue configurations outlined above. The angular densities of incident waves are assumed to be uniform in azimuth and Gaussian in elevation, as discussed in [16]. Under these assumptions, the angular densities assume the form

$$
P_{\xi}(\theta, \phi)=\frac{A_{\xi}}{2 \pi} \exp \left[-\frac{\left(\theta-m_{\xi}\right)^{2}}{2 \sigma_{\xi}^{2}}\right] \quad \theta<\pi / 2
$$

where $\xi=\theta$ or $\phi$ and $m_{\xi}$ and $\sigma_{\xi}$ denote the mean and standard deviation of the elevation angle for the $\hat{\xi}$ polarization. In this work, a value of $m_{\xi}=\pi / 2$ (horizontal plane) will be assumed for all computations. Also, based upon the findings in [1], [2] that incident multipath signals arrive at elevation angles less than $40^{\circ}$, we will use $\sigma_{\theta}=\sigma_{\phi}=40^{\circ}$. This is also consistent with other indoor measurements that indicate that multipath signals tend to arrive concentrated near the horizontal plane in the types of buildings considered during our measurement campaign [17]. The coefficient $A_{\xi}$ is determined from the property

$$
\int_{0}^{2 \pi} \int_{0}^{\pi} P_{\xi}(\theta, \phi) \sin \theta d \theta d \phi=1
$$

\section{B. Correlation Coefficient}

The correlation coefficient $\rho_{e}$ for the signal envelopes at the two antenna terminals provides a measure of the handset antenna diversity performance [10]. Unfortunately, no simple development exists for obtaining this parameter directly based upon the antenna gain characteristics and the average parameters of the multipath field. Therefore, a commonly used alternative approach which evaluates the signal correlation coefficient $\rho_{s}$ will be employed in this study. The envelope 


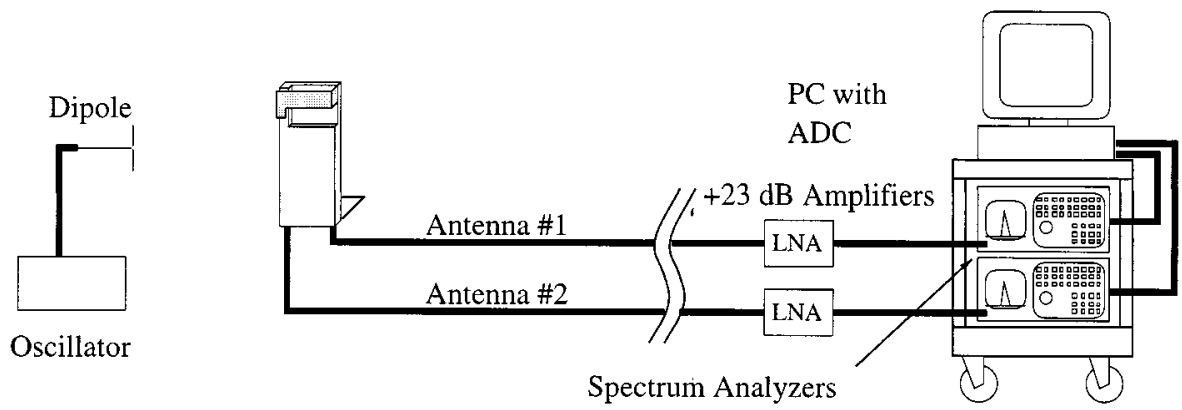

Fig. 2. Data acquisition platform consisting of two-branch receiver and remote transmitter.

correlation coefficient can then be approximated using the relation [10]

$$
\rho_{e} \approx\left|\rho_{s}\right|^{2}
$$

The details of this approach are provided in [8]. It has been shown that this relation is relatively accurate for urban environments where the multipath received signal is Rayleigh distributed. Therefore, it is interesting to compare the results of this simple approach to values obtained from the experimental measurements outlined below for the indoor environments assessed in this work.

\section{EXPERIMENTAL ASSESSMENT}

The values of MEG and $\rho_{e}$ obtained using the simulation procedures are best validated using measured results from scenarios that: 1) represent typical indoor propagation environments and 2) reflect realistic handset operation. This section describes the experimental techniques used to emulate these conditions.

\section{A. Measurement Platform}

The platform used to acquire measurements of different propagation environments and antenna diversity characteristics is shown schematically in Fig. 2. The transmitter consists of a Hewlett-Packard 8657A sweep oscillator at $915 \mathrm{MHz}$, which is connected to a vertically polarized, balun-fed dipole antenna. The receiving subsystem consists of two independent branches allowing simultaneous measurement of signals received on the two antennas. Each antenna signal is first pre-amplified using a low-noise amplifier providing $23 \mathrm{~dB}$ of gain. The amplified signal is then fed into a Hewlett-Packard 8590B spectrum analyzer operating in zero-span mode at the transmission frequency. The output voltage from the analyzer is sampled using a twochannel analog-to-digital converter (ADC) at a sample rate of $400 \mathrm{~Hz}$ per channel. During the measurement campaign, the system was calibrated by putting a known power level into each channel and properly weighting the ADC output to accurately represent the power level. A test performed by sweeping the input power from -40 to $-70 \mathrm{dBm}$ showed the system power reading to be linear to within $0.25 \mathrm{~dB}$.

\section{B. Measurement Campaign}

The data for this study were collected in two different buildings on the Brigham Young University campus. The

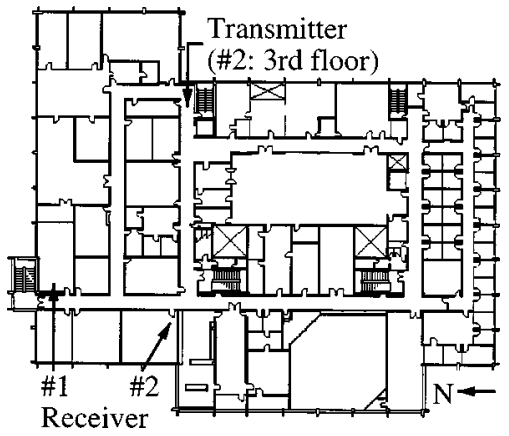

Fig. 3. Receiver and transmitter locations for sites 1 and 2 in the Clyde Engineering Building.

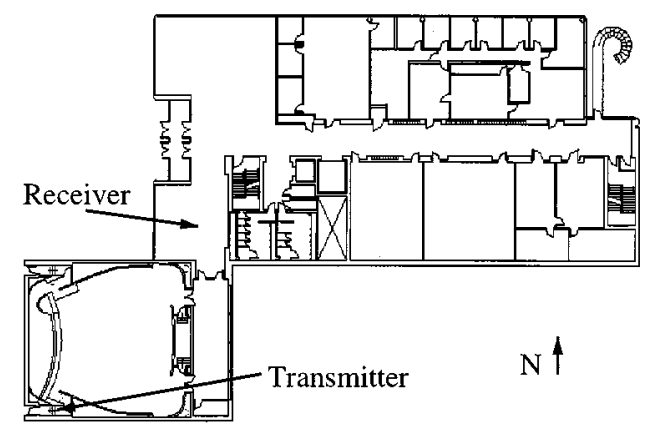

Fig. 4. Receiver and transmitter locations for site 3 in the Crabtree Technology Building.

first building, the four-story Clyde Engineering Building, consists of steel-reinforced concrete structural walls and cinder-block/brick partition walls. Two different measurement sites were used in this building, as shown in Fig. 3. For site 1, the transmitter and receiver were placed about $50 \mathrm{~m}$ apart on the building fourth floor. For site 2, the transmitter was placed on the third floor with the receiver (approximately 40 m away) on the fourth floor. The second building, the Crabtree Technology Building, contains steel-reinforced concrete structural walls and drywall/steel-stud partition walls. Only one measurement site (site 3) was used in this building, with the transmitter and receiver on the same floor and separated by a distance of approximately $30 \mathrm{~m}$, as depicted in Fig. 4.

The measurements at each site were performed by moving the transmitter over a circular path with a 3-m diameter. This 
pattern was chosen because: 1) it localizes the antenna position such that the average signal power remains relatively constant and 2) it lends repeatability to the measurements to allow comparison between operation with and without human tissue. In each case, the handset was moved at a rate of approximately 0.5 $\mathrm{m} / \mathrm{s}$. In measurements involving human operators, data was collected for two different subjects (adult males). It is noteworthy that recent findings indicate significant variability when measurements are taken across many different subjects [18] and, therefore, caution should be taken when using the limited set of measurements presented here. However, these results do show trends indicating the effect of the tissue on the antenna diversity performance that are useful for planning and estimation purposes.

\section{Cross-Polarization Discrimination Ratio}

To facilitate comparisons between simulations and measurements, it was first necessary to measure the XPR at each site. To accomplish this, channels 1 and 2 of the data acquisition platform were connected to vertically and horizontally polarized dipoles, respectively. These antennas were mounted $50 \mathrm{~cm}$ apart and $2 \mathrm{~m}$ above the ground on a cardboard cross member attached to a styrofoam mast. This structure was then affixed to the cart carrying the receiver. The data from each branch from two 50-s measurement sweeps, each containing 20000 envelope samples, were then averaged, and ratio of these results was taken to obtain XPR values of $X_{1}=X_{2}=5 \mathrm{~dB}$ at sites 1 and 2 and $X_{3}=1.5 \mathrm{~dB}$ at site 3 .

The separation between the antennas was used since it was difficult to mechanically mount the two dipoles in a colocated configuration. Additionally, the $50-\mathrm{cm}$ separation results in very little interference/mutual coupling between the two antennas. It should be emphasized that this configuration does not allow measurement of each field polarization at a single location. However, since the XPR is defined as the ratio of the average power in the vertical field component to that in the horizontal, this measurement should provide a good statistical sample of the field in the location under investigation, resulting in accurate estimation of the XPR.

\section{Mean Effective Gain}

To measure the MEG for each element, the handset under test was mounted on the cardboard cross-member and was oriented at $60^{\circ}$ from vertical. One of the handset antennas was connected to the receiver, while the second was terminated in a $50-\Omega$ load. The second receiver branch was connected to a vertically polarized half-wave dipole antenna positioned $50 \mathrm{~cm}$ from the handset on the cross member. Following completion of these measurements, the handset was removed from the mast and held by an operator, again at an angle of $60^{\circ}$, next to the right ear. The operator walked in front of the cart (approximately 150 $\mathrm{cm}$ from the dipole antenna) during the measurement.

From the acquired data, the MEG is computed using the formula [19]

$$
\mathrm{MEG}=\frac{\bar{P}_{A U T}}{\bar{P}_{d}}
$$

where $\bar{P}_{A U T}$ and $\bar{P}_{d}$ represent the time-average power received by the antenna under test and the dipole, respectively. It should be noted that in the computed result for MEG, the value is normalized by the computed MEG of a dipole antenna to allow comparisons between measured and simulated data.

\section{E. Correlation Coefficient}

For propagation environments where the received signal envelope is Rayleigh distributed and when the antennas are characterized by equal MEG, the envelope correlation coefficient allows estimation of diversity performance. To measure this parameter, the antennas were simultaneously connected to the receiver system. The handset (rotated $60^{\circ}$ from vertical) was either mounted on the mast or held by the user. For voltage envelopes $V_{1}$ and $V_{2}$ collected at antennas 1 and 2, respectively, the envelope correlation coefficient is given as

$$
\rho_{e}=\frac{E\left[\left(V_{1}-\bar{V}_{1}\right)\left(V_{2}-\bar{V}_{2}\right)\right]}{\sqrt{E\left[\left(V_{1}-\bar{V}_{1}\right)^{2}\right] E\left[\left(V_{2}-\bar{V}_{2}\right)^{2}\right]}}
$$

where $\bar{V}_{i}$ represents a time average of voltage envelope $V_{i}$.

\section{F. Diversity Gain}

In many practical environments, the received signal may not be Rayleigh distributed and the branch MEG values may be unequal. Furthermore, previous studies have shown [3] that measured values of $\rho_{e}$ can be negative, a possibility that is excluded in existing theoretical analyses [2]. In such nonideal circumstances, accurate determination of achievable diversity gain can only be performed through direct measurements in a variety of environments. In this work, measurement of the diversity gain was accomplished using the received signal envelope data collected for the correlation coefficient determination. This data was used to generate 1000-bin histograms of the received SNR, which were in turn normalized and integrated to produce cumulative density functions (CDFs) describing the SNR. By creating such statistical representations for signals received by each individual antenna as well as after the diversity combining circuitry, a direct assessment of the diversity gain can be performed. We now consider the formation of these histograms for both selection and equal-gain combining schemes.

1) Selection Combining: To determine the statistics for selection diversity combining, the data sequences representing the envelope power received by each antenna are combined by selecting the branch with the highest power. The noise power $N_{p}$ required in the determination of SNR was obtained by measuring the receiver power with the transmitter off. This procedure resulted in a value of $N_{p}=-104 \mathrm{dBm}$. The SNR sequence for selection combining is then given by

$$
\gamma_{\text {sel }}\left(t_{n}\right)=\frac{\max \left[P_{1}\left(t_{n}\right), P_{2}\left(t_{n}\right)\right]}{N_{p}}
$$

where $P_{1}$ and $P_{2}$ represent the power received by antennas 1 and 2 , respectively, and $t_{n}$ represents the discrete time of the sample.

2) Equal Gain Combining: Determining the SNR CDF for equal gain combining of the signals must be performed by first 
TABLE I

MEAsured (Meas) ANd Simulated (Sim) VALUes of MEG (dB)For INDIVIDUAL HANDSET ANTENNAS WITH AND Without THE USER PRESENT

\begin{tabular}{|c|c|c|c|c|c|c|}
\hline \multirow[b]{2}{*}{ Site } & \multirow[b]{2}{*}{ User } & & \multicolumn{2}{|c|}{ Handset \# 1} & \multicolumn{2}{|c|}{ Handset \# 2} \\
\hline & & & Mono & Mouth & PIFA & Mouth \\
\hline \multirow[t]{4}{*}{1} & $\overline{\mathrm{N}}$ & Meas & -2.32 & -2.90 & -2.66 & -2.01 \\
\hline & & Sim & -3.20 & -3.26 & -3.47 & -4.24 \\
\hline & $\mathrm{Y}$ & Meas & -5.44 & -5.67 & -6.55 & -5.83 \\
\hline & & Sim & -7.63 & -7.60 & -6.71 & -7.52 \\
\hline \multirow[t]{4}{*}{2} & $\mathrm{~N}$ & Meas & -1.27 & -2.35 & -2.00 & -0.89 \\
\hline & & Sim & -3.20 & -3.26 & -3.47 & -4.24 \\
\hline & Y & Meas & -7.17 & -5.46 & -7.06 & -7.33 \\
\hline & & Sim & -7.63 & -7.60 & -6.71 & -7.52 \\
\hline \multirow[t]{4}{*}{3} & $\mathrm{~N}$ & Meas & -0.82 & -0.99 & -1.81 & -1.61 \\
\hline & & Sim & -1.70 & -1.81 & -2.12 & -2.41 \\
\hline & $\mathrm{Y}$ & Meas & -4.89 & -4.10 & -5.61 & -5.95 \\
\hline & & Sim & -5.92 & -5.60 & -5.36 & -5.64 \\
\hline
\end{tabular}

adding the signal envelopes and using this resultant signal to form the SNR. Since the noise is assumed to be uncorrelated, the noise power from this combining strategy is simply twice that of a single branch. Mathematically, the resulting SNR sequence is expressed as

$$
\gamma_{e g}\left(t_{n}\right)=\frac{\left[\sqrt{P_{1}\left(t_{n}\right)}+\sqrt{P_{2}\left(t_{n}\right)}\right]^{2}}{2 N_{p}} .
$$

\section{RESULTS}

The following sections provide results obtained from both the simulation tools and the experimental platform discussed above. In the results shown, Handsets \#1 and \#2 refer the configurations in Fig. 1(a) and (b), respectively.

\section{A. Mean Effective Gain}

Table I provides a summary of the computed and measured MEG values for each of the different handset antennas in the three measurement sites. The results show reasonable agreement between the two sets of data. The discrepancies most likely arise from incorrect modeling assumptions in the MEG simulation model. Additionally, the values provided in Table I are similar to values reported in the literature for antenna/handset configurations of similar construction [11], [19], [20].

A notable observation concerning the data in Table I is the uniformity of MEG values for antennas on a single handset. With few exceptions, the results for different antennas on a handset remain within $1.5 \mathrm{~dB}$ and are generally within $0.5 \mathrm{~dB}$. The similarity of these values is important since both antennas must provide approximately equal mean SNR levels to the receiver in order to achieve high-diversity gain. Additionally, the data shows that the presence of the user's tissue reduces the MEG by between 3-6 dB. Such values are consistent with results from previous studies which indicate that between $50 \%$ and $70 \%$ of the power radiated by handsets held by a human operator is absorbed in the tissue [13]. Finally, it is noteworthy that the widest variability in MEG occurs at site 2, most likely due to the multipath structure observed there.
TABLE II

Measured (Meas) and Simulated (Sim) Values of Envelope CORRELATION COEFFICIENT FOR THE HANDSET BRANCHES WITH AND Without THE USER PRESENT

\begin{tabular}{lll|rr}
\hline \hline Site & User & & Handset \# 1 & Handset \# 2 \\
\hline \hline 1 & $\mathrm{~N}$ & Meas & 0.1262 & -0.0361 \\
& & Sim & 0.0377 & 0.1329 \\
& $\mathrm{Y}$ & Meas & 0.0758 & 0.1246 \\
& & Sim & 0.0006 & 0.0008 \\
\hline 2 & $\mathrm{~N}$ & Meas & 0.2866 & 0.0138 \\
& & Sim & 0.0377 & 0.1329 \\
& $\mathrm{Y}$ & Meas & 0.0908 & 0.0521 \\
& & Sim & 0.0006 & 0.0008 \\
\hline 3 & $\mathrm{~N}$ & Meas & 0.0965 & 0.1147 \\
& & Sim & 0.0614 & 0.1641 \\
& $\mathrm{Y}$ & Meas & 0.2719 & 0.2979 \\
& & Sim & 0.0015 & 0.0043 \\
\hline \hline
\end{tabular}

\section{B. Correlation Coefficient}

Table II shows measured and computed values of $\rho_{e}$ for the two different handsets at the three different measurements sites. Three different observations can be made concerning this data. First, both measurements and simulations show that correlation coefficients for each handset are very low (generally less than 0.2 ). This implies that both handsets will offer reasonable diversity performance. Second, while simulation shows that the operator tissue always reduces $\rho_{e}$, measurements indicate that the presence of the operator may actually increase the envelope correlation, likely due to narrowing of the antenna beam in the azimuthal plane because of strong coupling with the high-permittivity tissues in the head [11]. Finally, while the simulation approach precludes the possibility of negative correlation coefficients, Table II indicates that measured values of $\rho_{e}$ can be less than zero. However, it is notable that only one negative value appears in this table and that its magnitude is relatively small. It is conceivable that this negative value would become positive if more data were used in the computation.

\section{Diversity Gain}

Before assessing the diversity gain achievable with the handset configurations, it is interesting to determine the envelope statistics for signals received at each site. Fig. 5 plots the CDF for sites 1 and 2 for data measured with the top-mounted monopole of Fig. 1(a). The solid lines represent the best-fitting Rayleigh CDF for each case. These results show that the measured data are approximately Rayleigh distributed, suggesting the possibility of accurate estimation of diversity gain performance using theoretical analysis coupled with observed values of $\rho_{e}$. Results for site 3 are very similar to those observed for site 1 .

The diversity gain for each handset is assessed by comparing the CDF of the received SNR for the combined signal with that for a single branch. Because of the large volume of data collected, only representative curves for the actual CDF are included. However, averaged diversity gains at the 90, 95, and $99 \%$ reliability levels are tabulated for all cases. 


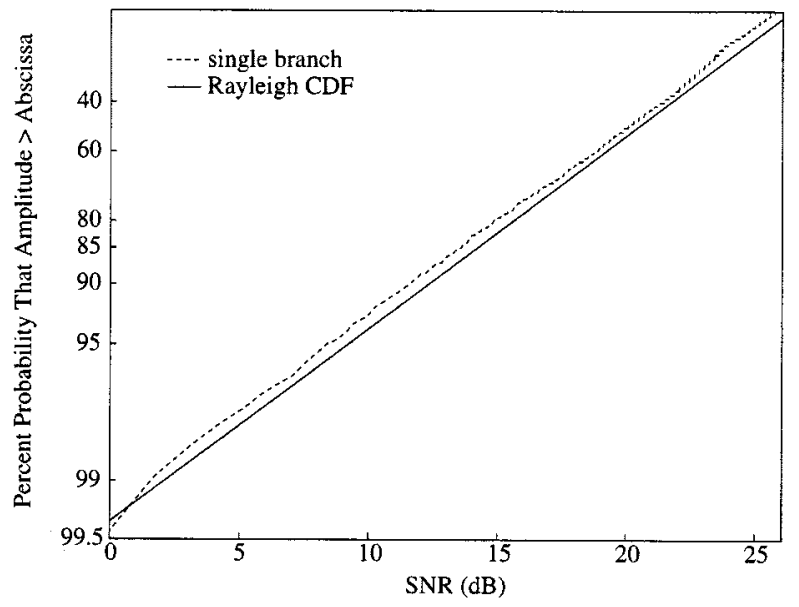

(a)

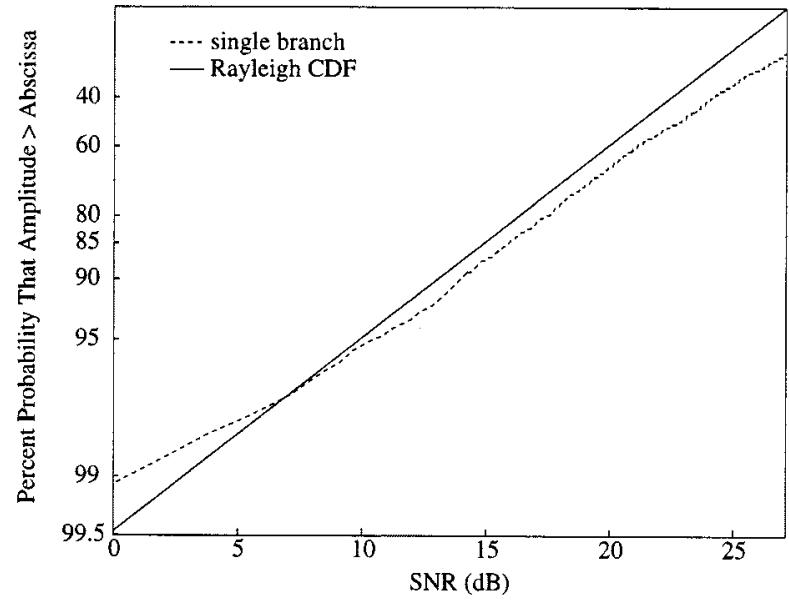

(b)

Fig. 5. CDF for signals received at (a) site 1 and (b) site 2 compared with the Rayleigh CDF.

We first consider the diversity performance of Handset \#1. Fig. 6(a) and (b) depict representative CDFs at site 1 for each branch alone as well as for the two combining strategies considered. The two figures are for the handset without and with the tissue, respectively. Diversity gain for this data is defined as the difference in SNR between the single-branch (highest of the two branch signals is chosen) and combined signals at a given probability level. Table III contains numerical gain values averaged from three different measurements for each site. The theoretical results are taken from curves in [2] for zero branch correlation. This data shows that at the $99 \%$ level, equal gain combining achieves a diversity gain between $9-11 \mathrm{~dB}$, while selection combining offers a gain of $8-10 \mathrm{~dB}$. It is also noteworthy that while the tissue tends to lower the antenna MEG, it has little impact on the system diversity gain. Finally, the results indicate that the theoretical gains obtained from ideal branches are very close to what has been observed in these measurements. Discrepancies in these values may arise from the nonequal effective antenna gains as well as the nonzero envelope correlation coefficients characterizing the handset antennas.

Fig. 7(a) and (b) depict representative CDFs for Handset \#2 at site 1 without and with tissue, respectively. Table IV tabulates the diversity gain at several different reliability levels. One no-

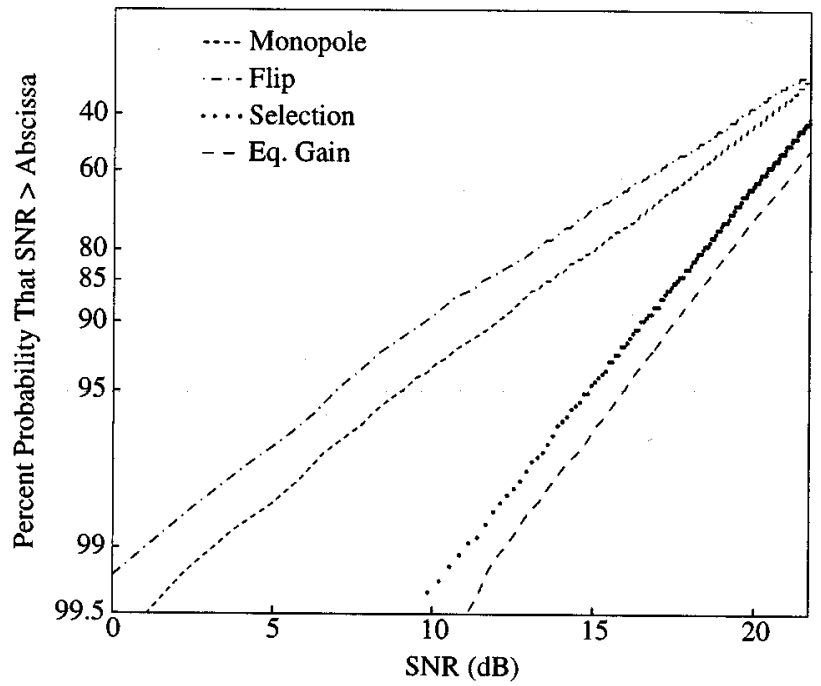

(a)

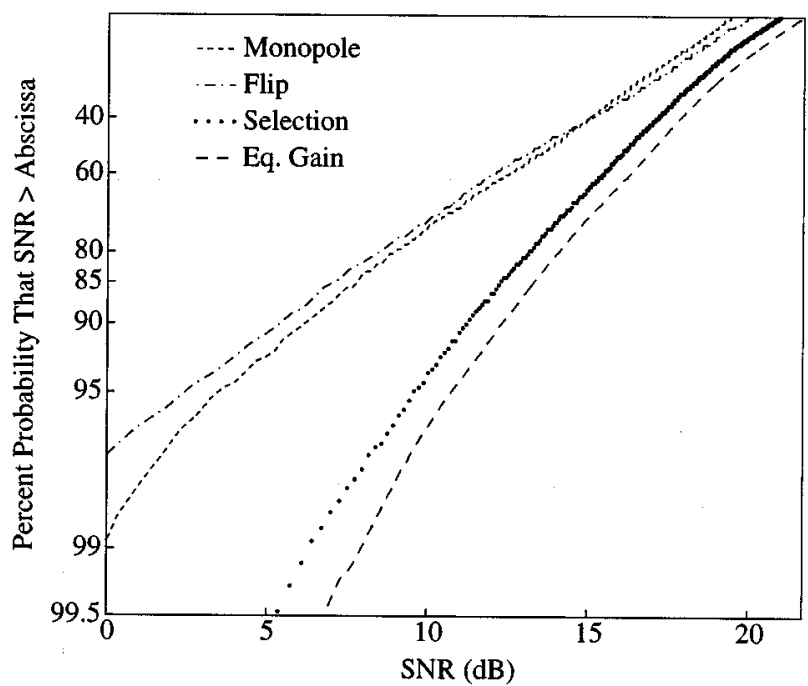

(b)

Fig. 6. CDF for signals received at site 1 for the handset in Fig. 1(a). Plots (a) and (b) represent data taken without and with the tissue, respectively.

TABLE III

AVERAGEd Diversity GaIN VAlues (dB) FOR HANDSET \#1

\begin{tabular}{ll|rrr|rrr}
\hline \hline & & \multicolumn{3}{|c}{ Selection } & \multicolumn{3}{c}{ Equal Gain } \\
Site & User & $90 \%$ & $95 \%$ & $99 \%$ & $90 \%$ & $95 \%$ & $99 \%$ \\
\hline \hline 1 & $\mathrm{~N}$ & 3.8 & 5.0 & 7.9 & 4.7 & 5.9 & 9.2 \\
& 1 & 4.7 & 5.6 & 6.6 & 5.8 & 6.7 & 8.1 \\
& 2 & 4.3 & 5.3 & 5.9 & 5.4 & 6.4 & 7.5 \\
\hline 2 & $\mathrm{~N}$ & 4.4 & 5.6 & 8.4 & 5.5 & 6.7 & 9.7 \\
& 1 & 4.4 & 5.4 & 5.4 & 5.4 & 6.5 & 7.0 \\
& 2 & 4.0 & 5.2 & 6.4 & 5.1 & 6.2 & 7.7 \\
\hline 3 & $\mathrm{~N}$ & 5.0 & 6.4 & 9.8 & 5.7 & 7.1 & 10.8 \\
& 1 & 4.6 & 5.5 & 9.1 & 5.5 & 6.4 & 10.0 \\
& 2 & 4.3 & 5.9 & 11.2 & 4.9 & 6.6 & 11.7 \\
\hline Theory & 5.6 & 6.7 & 10.0 & 5.5 & 7.3 & 10.9 \\
\hline \hline
\end{tabular}

table trend in this data is the variation in diversity gain values observed between sites 1 and 2 . This may be due to imbalances in the MEG between the two antenna elements caused by dif- 


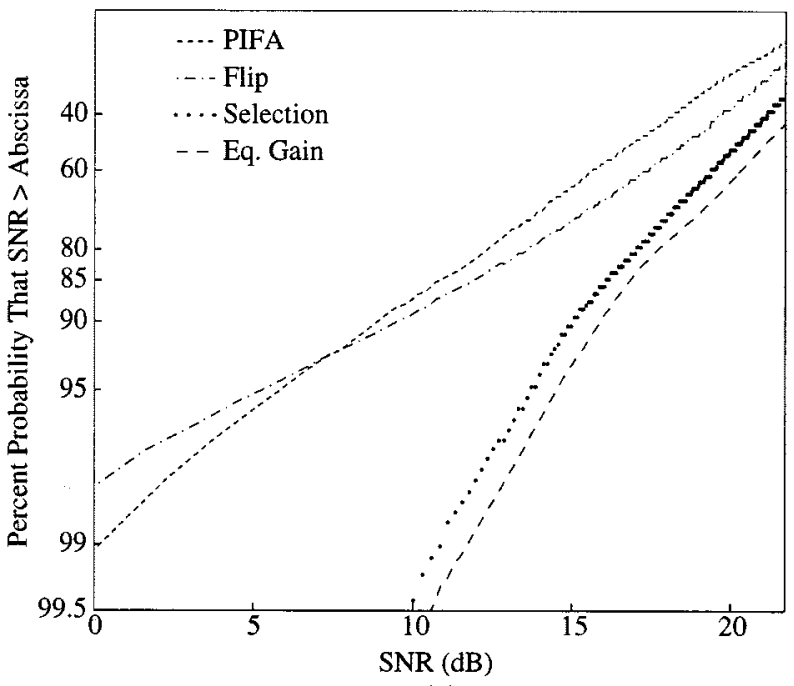

(a)

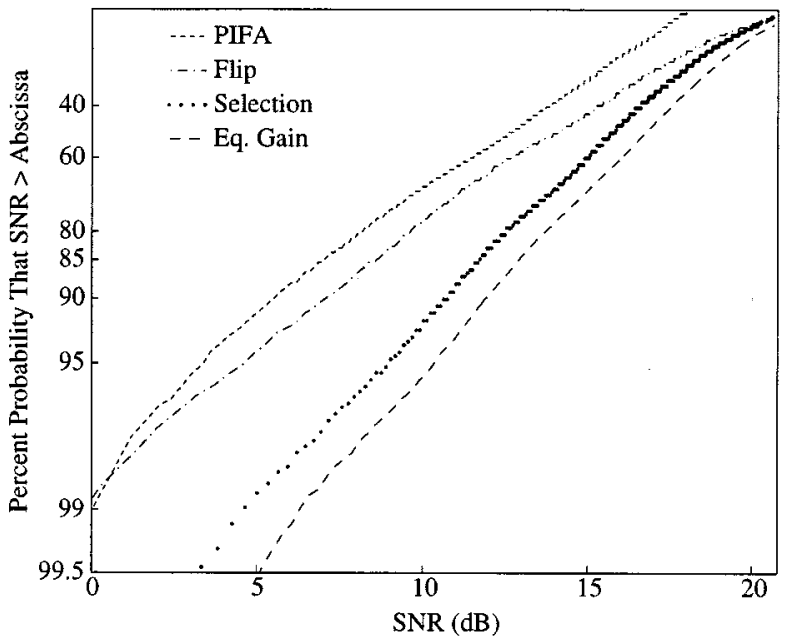

(b)

Fig. 7. CDF for signals received at site 1 for the handset in Fig. 1(b). Plots (a) and (b) represent data taken without and with the tissue, respectively.

TABLE IV

AVERAGED Diversity GaIN VALUES (dB) FOR HANDSET \#2

\begin{tabular}{ll|rrr|rrr}
\hline \hline & & \multicolumn{3}{|c}{ Selection } & \multicolumn{3}{c}{ Equal Gain } \\
Site & User & $90 \%$ & $95 \%$ & $99 \%$ & $90 \%$ & $95 \%$ & $99 \%$ \\
\hline \hline 1 & $\mathrm{~N}$ & 5.6 & 7.6 & 9.8 & 6.4 & 8.3 & 10.8 \\
& 1 & 4.1 & 4.7 & 5.6 & 5.3 & 6.0 & 7.3 \\
& 2 & 4.9 & 6.8 & 10.0 & 5.7 & 7.8 & 10.8 \\
\hline 2 & $\mathrm{~N}$ & 5.1 & 6.0 & 8.9 & 5.8 & 7.0 & 9.9 \\
& 1 & 4.4 & 4.7 & 4.5 & 5.5 & 5.9 & 6.2 \\
& 2 & 4.3 & 4.7 & 5.3 & 5.4 & 5.9 & 7.1 \\
\hline 3 & $\mathrm{~N}$ & 5.0 & 6.4 & 9.8 & 5.7 & 7.1 & 10.8 \\
& 1 & 5.7 & 6.9 & 11.5 & 6.4 & 7.6 & 12.5 \\
& 2 & 3.8 & 5.2 & 9.4 & 4.7 & 6.1 & 10.4 \\
\hline \multicolumn{2}{|c|}{ Theory } & 5.6 & 6.7 & 10.0 & 5.5 & 7.3 & 10.9 \\
\hline \hline
\end{tabular}

ferences in holding positions for the different users. As can be seen, this handset exhibits particularly high-diversity gains. In fact, in some instances the observed diversity gain is higher than the values predicted from the simple theoretical analysis.

\section{CONCLUSION}

This paper has presented the results of a computational and experimental study of the diversity performance of two dualantenna handsets operating in the indoor propagation environment. Particular attention was devoted to the effect of the operator tissue on the handset diversity performance. Computational results from the FDTD approach for MEG and envelope correlation coefficient were shown to agree relatively well with experimentally obtained data. Additionally, direct measurement of diversity gain was accomplished using a statistical analysis of the received signal envelopes. These results showed that the MEG of the individual branches were similar implying the potential for high-diversity gain. While the introduction of the tissue acted to lower the MEG by $3-5 \mathrm{~dB}$, it did not significantly change the similarity of MEG among the branches. Also, it was shown that the tissue exercised little influence on the envelope correlation coefficients and the achievable diversity gain. Of particular significance was the very low values of correlation coefficient observed. Experimental measurements of the diversity gain showed that theoretical predictions based upon zero branch correlation offer very good estimations of the gain available from dual-antenna configurations.

\section{REFERENCES}

[1] W. C. Y. Lee, Mobile Communications Engineering. New York: Wiley, 1982.

[2] W. C. Jakes Jr., Microwave Mobile Communications. New York: Wiley, 1974.

[3] R. G. Vaughan and J. B. Andersen, "Antenna diversity in mobile communications," IEEE Trans. Veh. Technol., vol. VT-36, pp. 149-172, Nov. 1987.

[4] R. G. Vaughan, "Polarization diversity in mobile communications," IEEE Trans. Veh. Technol., vol. 39, pp. 177-186, Aug. 1990.

[5] — "Signals in mobile communications: A review," IEEE Trans. Veh. Technol., vol. VT-35, pp. 133-145, Nov. 1986.

[6] K. Ogawa and T. Uwano, "A diversity antenna for very small $800-\mathrm{MHz}$ band portable telephones," IEEE Trans. Antennas Propagat., vol. 42, pp. 1342-1345, Sept. 1994.

[7] J. S. Colburn, Y. Rahmat-Samii, M. A. Jensen, and G. J. Pottie, "Evaluation of personal communications dual antenna handset diversity performance," IEEE Trans. Veh. Technol., vol. 37, pp. 737-746, Aug. 1998.

[8] M. A. Jensen and Y. Rahmat-Samii, "Performance analysis of antennas for hand-held transceivers using FDTD," IEEE Trans. Antennas Propagat., vol. 42, pp. 1106-1113, Aug. 1994.

[9] J. Lemieux, M. S. El-Tanany, and H. M. Hafez, "Experimental evaluation of space/frequency/polarization diversity in the indoor wireless channel," IEEE Trans. Veh. Technol., vol. 40, pp. 569-573, Aug. 1991.

[10] J. N. Pierce and S. Stein, "Multiple diversity with nonindependent fading," IRE Proc., pp. 89-104, Jan. 1960.

[11] G. F. Pedersen and S. Skjaerris, "Influence on antenna diversity for a handheld phone by the presence of a person," in Proc. 47th IEEE Veh. Technol. Conf., vol. 3, Phoenix, AZ, May 1997, pp. 1768-1772.

[12] A. Taflove, Computational Electrodynamics: The Finite-Difference Time-Domain Method. Boston, MA: Artech House, 1995.

[13] M. A. Jensen and Y. Rahmat-Samii, "EM interaction of handset antennas and a human in personal communications," Proc. IEEE, vol. 83, pp. 7-17, Jan. 1995.

[14] J. Toftgârd, S. N. Hornsleth, and J. B. Andersen, "Effects on portable antennas of the presence of a person," IEEE Trans. Antennas Propagat., vol. 41, pp. 739-746, June 1993.

[15] J. B. Andersen and F. Hansen, "Antennas for VHF/UHF personal radio: A theoretical and experimental study of characteristics and performance," IEEE Trans. Veh. Technol., vol. VT-26, pp. 349-357, Nov. 1977.

[16] T. Taga, "Analysis for mean effective gain of mobile antennas in land mobile radio environments," IEEE Trans. Veh. Technol., vol. 39, pp. 117-131, May 1990. 
[17] M. A. Jensen, Q. H. Spencer, A. L. Swindlehurst, and B. D. Jeffs, "Measurement and modeling of temporal and spatial indoor multipath characteristics," in 1999 IEEE AP-S Int. Symp. Dig., vol. 1, Orlando, FL, July 1999, pp. 388-391.

[18] G. F. Pedersen, J. O. Nielsen, K. Olesen, and I. Z. Kovacs, "Measured variation in performance of handheld antennas for a large number of test persons," in Proc. 48th IEEE Veh. Technol. Conf., vol. 1, Ottawa, Canada, May 1998, pp. 505-509.

[19] M. Murase, Y. Tanaka, and H. Arai, "Propagation and antenna measurements using antenna switching and random field measurements," IEEE Trans. Veh. Technol., vol. 43, pp. 537-541, Aug. 1994.

[20] H. Arai, N. Igi, and H. Hanaoka, "Antenna-gain measurement of hand held terminals at $900 \mathrm{MHz}$," IEEE Trans. Veh. Technol., vol. 46, pp. 537-543, Aug. 1997.

Bruce M. Green received the B.S. and M.S. degrees from Brigham Young University (BYU), Provo, UT, in 1996 and 1997, respectively. He is currently working toward the Ph.D. degree at Cornell University, Ithaca, NY.

He has authored or coauthored ten technical publications and has one patent pending. At BYU his research focused on the evaluation and modeling of personal communication handset antennas systems in indoor propagation environments. His research at Cornell focuses on the modeling, design, and fabrication of gallium nitride devices and integrated circuits for high-power high-frequency applications.

Mr. Green received a National Science Foundation Graduate Research Fellowship in 1996. In 1998 he received an IEEE MTT-S Graduate Fellowship. In 1999 he received a Schlumberger Foundation Fellowship. He is a member of Tau Beta Pi and Eta Kappa Nu.

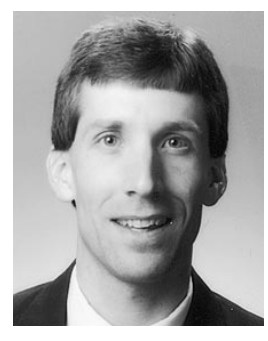

Michael A. Jensen (S'93-M'95) received the B.S. (summa cum laude) and M.S. degrees in electrical engineering from Brigham Young University (BYU), Provo, UT, in 1990 and 1991, respectively, and the Ph.D. degree in electrical engineering from the University of California at Los Angeles (UCLA), in 1994.

From 1989 to 1991 he was a Graduate Research Assistant in the Lasers and Optics Laboratory, BYU. From 1991 to 1994 he was a Graduate Student Researcher in the Antenna Laboratory, UCLA. Since 1994, he has been an Assistant Professor in the Electrical and Computer Engineering Department, BYU. His main research interests include radiation and propagation for personal communications, radar remote sensing, numerical electromagnetics, and optical fiber communications.

Dr. Jensen received a National Science Foundation Graduate Fellowship in 1990. He is a member of Eta Kappa Nu and Tau Beta Pi. 